\title{
Ketofol:A Combination of Ketamine and Propofol
}

\begin{abstract}
Ketofol is a combination of ketamine and propofol. To date, there is significant interest in ketofol as an agent for procedural sedation and analgesia. The combination of propofol and ketamine has several benefits in the terms of hemodynamic stability, absence of respiratory depression, post-operative analgesia and recovery. This combination can be mixed in the same syringe or administered independently in the two separate syringes. It can be administered as a bolus or as a continuous infusion for longer procedures. Ketaminepropofol combinations in the different ratios have been studied before. The optimal mixture and dosing of ketamine and propofol has yet to be determined. This short review will highlight recent clinical supports the therapeutic utility of the combination of ketamine and Propofol.
\end{abstract}

Volume I Issue 5 - 2014

\section{Somchai Amornyotin}

Department of Anesthesiology and Siriraj GI Endoscropy

Center, Faculty of Medicine Siriraj Hospital, Thailand

\begin{abstract}
Correspondence: Somchai Amornyotin, Department of Anesthesiology and Siriraj GI Endoscopy Center, Faculty of Medicine Siriraj Hospital, Mahidol University, Bangkok 10700, Thailand,Tel 66-2-419-7990, Email somchai.amo@mahidol.ac.th
\end{abstract}

Received: October 24, 2014 | Published: November 18, 2014

Keywords: ketofol; ketamine; propofol; efficacy; safety

\section{Introduction}

Sedative and analgesic agents are frequently used for procedural sedation. Titration of anesthetic doses should be done cautiously and the patients should be continuously monitored. So far, an ideal intravenous anesthetic agent is none ${ }^{1,2}$. A combination of ketamine and propofol has several ideal anesthetic properties. Several clinical studies of the use of ketofol have been evaluated. To date, there is significant interest in ketofol as an agent for procedural sedation. This report highlights on the combination of ketamine and propofol (Ketofol).

\section{Ketofol}

Ketofol is the combination of ketamine and propofol in various concentrations. It commonly used for several procedures. Ketamine, a neuroleptic anesthetic agent, works on thalamocortical and limbic N-methyl-D-aspartate (NMDA) receptors. ${ }^{1,2}$ It can be given through intravenous or intramuscular routes. Ketamine stimulates the cardiorespiratory system. A direct effect increases cardiac output, arterial blood pressure, heart rate and central venous pressures. Therefore, it is a valuable agent for hypotensive or hypovolemic patients, but a less desirable agent in patients with ischemic heart disease or raised pulmonary vascular pressure. However, ketamine induces psychomimetic activity and emergence reactions in up to $30 \%$ of patients. In contrast, propofol, a sedative, hypnotic and anesthetic agent, is also an antagonist at N-methyl-D-aspartate receptors. ${ }^{1,2}$ However, propofol has a narrow therapeutic range and risks of cardiovascular depression. ${ }^{3}$ It is legally used by anesthetic personnel in many countries. The use of propofol by nonanesthesiologists is controversial. ${ }^{4,5}$ However, propofol-based sedation is safe and highly effective. Mild respiratory adverse events occur frequently and major complications may happen rarely. Additionally, the adverse events do not occur more frequently compared to other sedation regimens. ${ }^{6-8}$ As a result, the combination of these two drugs has several advantages.

\section{Ketofol mixtures}

The Propofol-ketamine mixtures have been used for procedural sedation. Several studies had been published. The study of Wang and colleagues investigated the propofol-ketamine mixtures in the ratios of 2:1, 3:1 and 4:1 compared with the combination of propofol and fentanyl as well as the Propofol alone. The study demonstrated that ketofol was safe and effective as the combination of propofol and fentanyl combination. The ratios of $2: 1,3: 1$ and $4: 1$ were very effective for the procedure. The efficacy of sedation, recovery and discharge time in the ratios of 3:1 and 4:1 mixtures of ketofol presented comparable. Additionally, the incidence of respiratory depression and postprocedural dizziness in the ratio of 4:1 (40 mg of ketamine and $160 \mathrm{mg}$ of propofol in a $20 \mathrm{~mL}$ syringe) was a relatively lower than in the other ratios of ketofol. Additional doses of propofol in the propofol only group were also significantly higher. ${ }^{9}$

Dal et al., ${ }^{10}$ compared the effectiveness and safety of the ketofol and the combination of ketamine and midazolam for procedural sedation in the endobronchial ultrasound-guided needle aspiration. The 60 adult patients were randomized into the two groups. Group KP was given $0.125 \mathrm{mg} / \mathrm{kg}$ i.v. ketamine and propofol mixture (ketofol) and injection of $0.25 \mathrm{mg} / \mathrm{kg}$ each. Group KM was given $0.25 \mathrm{mg} /$ $\mathrm{kg}$ i.v. ketamine and $0.05 \mathrm{mg} / \mathrm{kg}$ i.v. midazolam. Procedural sedation was maintained with additional doses of ketofol $0.125 \mathrm{mg} / \mathrm{kg}$ and ketamine $0.25 \mathrm{mg} / \mathrm{kg}$ in Group KP and Group KM, respectively. The result of their study demonstrated that HR in the $10^{\text {th }}$ min and Ramsay Sedation Score (RSS) in the $35^{\text {th }}$ min in group KP were significantly lower than in group KM. Additionally, the recovery time in group KP was significantly shorter than group KM. However, there were no significantly differences in the consumption of the agents, oxygen saturation, respiratory rate, RSS value and the severity of cough as well as the satisfaction of physician and the patients between the two groups. The authors concluded that ketofol was effective and safe for sedation in the endobronchial ultrasound-guided needle aspiration procedure. No serious adverse events were observed.

Phillips et al., ${ }^{11}$ compared ketamine and propofol with propofol alone in 28 patients underwent procedural sedation in the emergency department. The intravenous ketamine doses ranged from 0.5 to $1 \mathrm{mg} / \mathrm{kg}$, with propofol $0.75 \mathrm{mg} / \mathrm{kg}$. The comparator intravenous propofol doses ranged from 0.5 to $1.5 \mathrm{mg} / \mathrm{kg}$. The primary outcome was adequacy of sedation measured using the bispectral index scale (BIS). The propofol-ketamine group presented less of a difference in BIS between baseline and goal sedation. The authors recommended that adequate sedation with the combination of propofol and ketamine was completed without the need for deep sedation compared with the propofol alone. Safety outcomes revealed significantly less reduction in blood pressure in the propofol-ketamine group. Neither group experienced respiratory depression or a significant difference in length of sedation.

Another previous study also compared the quality of sedation and side effects of two different ratios of ketofol in 60 pediatric patients under lumbar puncture or bone marrow aspiration. They divided the 
patients into the 1:2 and the 1:3 ratios of ketofol. The results confirmed that the 1:3 ratio of ketofol had lower psychological side effects and shorter recovery time than the 1:2 ratio of ketofol. However, the quality of sedation, the total dose of drug and respiratory and hemodynamic parameters were comparable in both groups. ${ }^{12}$

A randomized, double-blind, placebo-controlled trial compared the frequency of respiratory depression in 98 adults and 93 children receiving a propofol-ketamine combination and propofol alone for procedural sedation in the emergency department. ${ }^{13}$ All patients received an intravenous dose of fentanyl $0.5-1.0 \mathrm{meg} / \mathrm{kg} 5 \mathrm{~min}$ before sedation. Patients were given either ketamine $0.5 \mathrm{mg} / \mathrm{kg}$ or placebo over $1 \mathrm{~min}$, followed by a dose of propofol $1 \mathrm{mg} / \mathrm{kg}$ over $2 \mathrm{~min}$. Bolus doses of propofol $0.5 \mathrm{mg} / \mathrm{kg}$ were given as needed to maintain a Colorado Behavioral Numerical Pain Scale of 0 (restful, no facial expression). The depth of sedation level in both groups was similar. There was no significant difference in respiratory depression between the two groups. However, there was a significant difference in the overall satisfaction of the quality of the sedation, with $95 \%$ of physicians being satisfied with propofol-ketamine compared to $65 \%$ satisfied with propofol alone. There were no reported occurrences of emergence reactions or other adverse drug events.

Coulter et al., ${ }^{14}$ evaluated the Ketofol in the different ratios for general anesthesia in the pediatric patients. The drug concentration and the anesthesia profiles of ketofol were also studied. The authors concluded that infusion of ketofol would prolong recovery period if the infusion rate was not decreased. They suggested an optimal ratio of ketamine to propofol of 1:5 for a 30 min-anesthesia, and 1:6.7 for a $90 \mathrm{~min}$-anesthesia. The same authors evaluated the ketofol in the different ratios for procedural sedation in the healthy pediatric patients. They suggested that a 1:3 ratio of ketamine and propofol was the best combination for the intermittent dosing. An optimal dose of ketofol for pediatric patients was $0.1 \mathrm{~mL} / \mathrm{kg}$ in the beginning followed by $0.05 \mathrm{~mL} / \mathrm{kg}$ at $2 \mathrm{~min}$ and then $0.025 \mathrm{~mL} / \mathrm{kg}$ for the subsequent doses. Consequently, an optimal dose of ketofol for adult patients was $0.05 \mathrm{~mL} / \mathrm{kg}$ followed by $0.025 \mathrm{~mL} / \mathrm{kg}$ for the subsequent doses. Furthermore, the mixing ratio greater than $1: 3$ resulted in prolong of recovery. ${ }^{15}$

Another prospective case series was conducted the use of propofolketamine in the emergency department. ${ }^{16}$ The propofol-ketamine was administered in a 1:1 ratio mixed in the same syringe (median dose $0.75 \mathrm{mg} / \mathrm{kg}$ ). All patients could receive opioids before the procedure at the discretion of the physician. Of the 114 procedures attempted, $96 \%$ required no adjunct medications to complete the procedure. The median recovery time for propofol-ketamine was $15 \mathrm{~min}$. Apnea occurred in three patients? The bag-valve-mask ventilation was required for one, while two patients required repositioning, stimulation and supplemental oxygen. A fourth patient experienced recovery agitation and required midazolam.

Recently, Nalini et al., ${ }^{17}$ studied 60 patients, ASA physical status I who underwent puerperal sterilization. All patients were randomized into ketamine-propofol infusion in a concentration of $8 \mathrm{mg} / \mathrm{ml}$ each (group PK) and fentanyl $2 \mathrm{mcg} / \mathrm{kg}$ followed by a continuous infusion of $8 \mathrm{mg} / \mathrm{ml}$ propofol (group PF). In both groups, the infusion was started at $300 \mathrm{~mL} / \mathrm{h}$ till the patient lost consciousness. After that, the rate was set at $0.75 \mathrm{~mL} / \mathrm{kg} / \mathrm{h}$ for group PK and at $1.5 \mathrm{~mL} / \mathrm{kg} / \mathrm{h}$ for group PF, and was reduced to $0.5 \mathrm{~mL} / \mathrm{kg} / \mathrm{h}$ for group PK and $1 \mathrm{~mL} /$ $\mathrm{kg} / \mathrm{h}$ for group PF after $10 \mathrm{~min}$. The results demonstrated that the reduction of systolic and diastolic blood pressure as well as oxygen saturation in group PK was significantly lower than in group PF. The patients in group PK had also better surgical and recovery conditions.
The authors concluded that this combination technique (ketofol) is safe and may be an alternative regimen to the combination of propofol and fentanyl.

A Ketofol is also frequently used to achieve sedation and analgesia during endoscopic procedures. The author compared and evaluated the clinical efficacy of the ketofol and propofol alone when each regimen is used as sedative agents for colonoscopy. The 194 patients were randomized into two groups. The 97 patients in group PK received propofol and ketamine and 97 patients in group P received propofol and normal saline for sedation. All patients were premedicated with $0.02-0.03 \mathrm{mg} / \mathrm{kg}$ of midazolam. All endoscopic procedures were completely successful. The mean total dose of propofol in group PK and $P$ were $6.98 \pm 2.90 \mathrm{mg} / \mathrm{kg} / \mathrm{h}$ and $7.73 \pm 3.45 \mathrm{mg} / \mathrm{kg} / \mathrm{h}$, respectively. Mean total dose of ketamine in group PK was $1.49 \pm 0.61 \mathrm{mg} / \mathrm{kg} / \mathrm{h}$. There were no significant differences in patient tolerance, discomfort during insertion, patient and endoscopist satisfaction, hemodynamic responses, procedural pain, recovery time and recovery score. Overall, cardiovascular and respiratory adverse events were not significantly different between the two groups. These adverse events were transient and easily treated with no sequelae. ${ }^{18}$

Interestingly, Ketofol can be used for electroconvulsive therapy. Kayhan and colleagues evaluated the effect of a ketamine-propofol combination (ketofol) for electroconvulsive therapy (ECT) on seizure activity, hemodynamic response and recovery parameters. The 24 patients underwent a total of 144 ECT procedures were randomized into $1 \mathrm{mg} / \mathrm{kg}$ ketofol $(0.5 \mathrm{mg} / \mathrm{kg}$ propofol and $0.5 \mathrm{mg} / \mathrm{kg}$ ketamine $)$ and $1 \mathrm{mg} / \mathrm{kg}$ propofol $1 \%$ for anesthesia induction. Seizure duration and quality, hemodynamic data, recovery parameters and side effects were analyzed between the two groups. The seizure durations in both groups were similar. Post-ictal suppression index in the propofol group was lower than in the ketofol group. The heart rate and mean arterial pressure in the propofol group were also lower than in the ketofol group. Time to obeying commands was longer in the ketofol group. The undesirable psychological reactions were none in the ketofol group. The authors concluded that ketofol could be an optional technique for electroconvulsive therapy. ${ }^{19}$

\section{Conclusion}

Ketofol is a combination of ketamine and propofol. It is an agent of choice for various procedures. The combination of propofol and ketamine has several benefits because of hemodynamic stability, lack of respiratory depression, good recovery and potent post-procedural analgesia. The safety and efficacy of ketofol as a sedoanalgesic agent are depended on the dose and the ratio of the mixture. Therefore, ketofol should be an ideal combination drug for procedural sedation.

\section{Acknowledgments}

None.

\section{Conflicts of interest}

Author declares there are no conflicts of interest.

\section{Funding}

None.

\section{References}

1. Amornyotin S. Sedative and analgesic drugs for gastrointestinal endoscopic procedure. J Gastroenterol Hepatol Res. 2014;3(7):11331144 . 
2. Pandit JJ. Intravenous anesthetic agents. Anesth Intens Care Med 2011;12(4):144-150.

3. Amornyotin S. Sedation-related complications in gastrointestinal endoscopy. World J Gastrointest Endosc. 2013;5(11):527-533.

4. Tan G, Irwin MG. Recent advances in using propofol by nonanesthesiologists. F 1000 Med Reports. 2010;2:79.

5. Vargo JJ, Cohen LB, Rex DK, et al. Position statement: nonanesthesiologist administration propofol for GI endoscopy. Gastroenterology . 2009;137(6):2161-2167.

6. Amornyotin S, Aanpreung P, Prakarnrattana U, et al. Experience of intravenous sedation for pediatric gastrointestinal endoscopy in a large tertiary referral center in a developing country. Pediatr Anesth. 2009;19(8):784-791.

7. Amornyotin S, Kongphlay S. Esophagogastroduodenoscopy procedure in sick pediatric patients: a comparison between deep sedation and general anesthesia technique. J Anesth Clin Res. 2012;3(1):185.

8. Amornyotin S, Aanpreung P. Clinical effectiveness of an anesthesiologistadministered intravenous sedation outside of the main operating room for pediatric upper gastrointestinal endoscopy in Thailand. International Journal of Pediatrics ID. 2010;748564.

9. Wang Y, Jiang X, Pang L, et al. A randomized double-blind controlled study of the efficacy of ketofol with propofol-fentanyl and propofol alone in termination of pregnancy. Afr J Pharm Pharmacol. 2012;6(34):2510 2514

10. Dal T, Sazak H, Tunc M, et al. A comparison of ketamine-midazolam and ketamine-propofol combinations used for sedation in the endobronchial ultrasound guided transbronchial needle aspiration: a prospective, single blind, randomized study. J Thorac Dis. 2014; 6(6):742-751.
11. Phillips W, Anderson A, Rosengreen M, et al. Propofol versus propofol/ ketamine for brief painful procedures in the emergency department: clinical and bispectral index scale comparison. J Pain Palliat Care Pharmacother. 2010;24(5):349-355.

12. Ghadami Yazdi A, Ayatollahi V, Hashemi A, et al. Effect of two different concentrations of propofol and ketamine combinations (Ketofol) in pediatric patients under lumbar puncture or bone marrow aspiration. Iranian J Pediatr Hematol Oncol. 2013;3(1):187-192.

13. David H, Shipp J. A randomized controlled trial of ketamine/propofol versus propofol alone for emergency department procedural sedation. Ann Emerg Med. 2011;57(5):435-441.

14. Coulter FLS, Hannam JA, Anderson BJ. Ketofol simulations for dosing in pediatric anesthesia. Pediatr Anesth. 2014;24(8):806-812.

15. Coulter FLS, Hannam JA, Anderson BJ. Ketofol dosing simulations for procedural sedation. Pediatr Emerg Care. 2014;30(9):621-630.

16. Willman EV, Andolfatto G. A prospective evaluation of 'ketofol' (ketamine/propofol combination) for procedural sedation and analgesia in the emergency department. Ann Emerg Med . 2007;49(1):23-30.

17. Nalini KB, Cherian A, Balachander H, et al. Comparison of propofol and ketamine versus propofol and fentanyl for puerperal sterilization, a randomized clinical trial. J Clin Diag Res. 2014;8(5):GC01-GC04.

18. Amornyotin S, Chalayonnawin W, Kongphlay S. Clinical efficacy of the combination of propofol and ketamine (ketofol) for deep sedation for colonoscopy. Gut. 2012;61(Suppl 2):A339-A340.

19. Kayhan GE, Yucel A, Colak YZ, et al. Ketofol (mixture of ketamine and propofol) administration in electroconvulsive therapy. Anaesth Intensive Care. 2012;40(2):305-310. 